\title{
ASTEROIDS
}

\section{Solar supercatastrophes}

Astrophys. J. (in the press); preprint at https:// arxiv.org/abs/2001.09839

Observations have shown a distinct dearth of asteroids or comets with perihelion very close to the Sun. While it is expected that objects falling into the Sun are destroyed, this lack of asteroids near the Sun but far from the solar surface is puzzling. It has been proposed that asteroids get destroyed when their perihelion is closer than $\sim 15$ solar radii, $R_{\odot}$ (Granvik et al. Nature 530, 303-306; 2016), but the cause of this supercatastrophic disruption is unclear. Paul Wiegert and collaborators propose that it is due to erosion by near-Sun meteoroids.

Wiegert et al. study and review the populations of various Sun-grazing objects using data from different surveys, including comets observed by the SOHO coronagraph, asteroids and meteoroids down to a size of a few millimetres. Just one body (asteroid (467372) 2004 LG) challenges the supercatastrophic theory by spending a significant amount of time within $15 R_{\odot}$ from the Sun. Interestingly, active asteroid (3200) Phaethon grazes the supercatastrophic limit (its perihelion is $\sim 30 R_{\odot}$ ), and it might represent a slow case of disruption. Only the population of millimetre-sized meteoroids, extrapolated from radar observations, is unaffected by heliocentric distance, and thus becomes the dominant source of objects near the Sun. The authors suggest that high-energy impacts from these small particles grind all bodies to almost complete destruction, without passing through an intermediate phase of metre-sized pieces.

While additional studies need to be undertaken in order to discriminate meteoroid erosion from competing processes, this theory can explain some observed phenomena, such as the sudden brightening and similarity of the lightcurves of near-Sun comets when they get close to our star.

Luca Maltagliati

Published online: 25 February 2020

https://doi.org/10.1038/s41550-020-1047-x 